\title{
NURBS Function Closed-Loop Mapping Trajectory Planning of Serial Robotic Plasma Cladding for Complex Surface Coatings
}

\section{Zhaoqin Wang}

Lanzhou University of Technology

Yu Shi ( $\square$ lanzhou_rail_1@163.com)

Lanzhou University of Technology

\section{Xiaorong Wang}

Lanzhou University of Technology

\section{Research Article}

Keywords: NURBS function mapping, NURBS surface coating, trajectory planning, plasma cladding, NURBS interpolation.

Posted Date: January 20th, 2022

DOI: https://doi.org/10.21203/rs.3.rs-1257603/v1

License: (c) (i) This work is licensed under a Creative Commons Attribution 4.0 International License. Read Full License 


\title{
NURBS function closed-loop mapping trajectory planning of serial robotic plasma cladding for complex surface coatings
}

\author{
Zhaoqin Wang ${ }^{a, b, c}$, Yu Shi ${ }^{a, *}$, Xiaorong Wang ${ }^{b, c}$ \\ ${ }^{a}$ State Key Laboratory of Advanced Processing and Recycling of Nonferrous Metals, Lanzhou University of \\ Technology, Lanzhou 730050, China \\ ${ }^{b}$ School of Mechatronic Engineering, Lanzhou Jiaotong University, Lanzhou 730010, China \\ ${ }^{c}$ Key Laboratory of System Dynamics and Reliability of Rail Transport Equipment of Gansu Province, Lanzhou \\ Jiaotong University, Lanzhou 730010, China \\ *Corresponding author: Yu Shi. Tel./Fax: +86 9312973563. \\ E-mail address: lanzhou_rail_1@163.com
}

\begin{abstract}
In order to solve the problem of the trajectory planning of robotic plasma cladding for complex surface coating, the concept of NURBS function open/closed-loop mapping is proposed in this work to explore the new approach of the trajectory planning for complex NURBS surfaces. The trajectory planning is carried out by a 2D-NURBS curve $\boldsymbol{C}(u)$ on a $2 \mathrm{D}$ plane, which is mapped on a predefined 3D-NURBS surface $S(u, v)$ using the NURBS surface function to form a 3D mapping curve named NURBS function mapping (NURBS-FM) curve. Using the fixed step (FS) interpolation, the equal chord length (ECL) interpolation, the equal arc length (EAL) interpolation and the equal bow height (EBH) interpolation, etc., a complex curve can be interpolated. The FS/ECL/EAL/EBH can be defined as constraint. Depending on where the constraint is applied, the NURBS-FM can be divided into open-loop mapping and closed-loop mapping. The NURBS function open-loop mapping
\end{abstract} (NURBS-FOLM) is carried out along the route of $u \rightarrow \boldsymbol{C}(u) \rightarrow \boldsymbol{S}(u, v)$, while the NURBS function closed-loop mapping (NURBS-FCLM) along the route of $u \rightarrow \boldsymbol{C}(u) \rightarrow \boldsymbol{S}(u, v) \rightarrow u \rightarrow \ldots$ The constraint is applied to the 2D-NURBS curve in NURBS-FOLM, while to the 3D NURBS-FM curve in NURBS-FCLM. The NURBS-FOLM and NURBS-FCLM can ensure that the interpolation points on the 2D-NURBS curve and 3D NURBS-FM curve have the ECL/EAL/EBH characteristics, respectively. When the 3D NURBS-FM curve is regarded as plasma cladding trajectory, the NURBS-FCLM can provide engineers with a new ECL/EAL/EBH interpolation method for cladding trajectory. The simulation and experiment verify the NURBS-FCLM is feasible and effective. With the increasing application of complex surface protective coatings prepared by plasma cladding, the NURBS-FCLM has broad application prospects inevitably.

Keywords: NURBS function mapping; NURBS surface coating; trajectory planning; plasma cladding; NURBS interpolation.

\section{Introduction}

NURBS (Non-Uniform Rational B-Splines) is a powerful mathematical model to express complex curves and surfaces due to its generality, and has been widely used in contemporary Computer-Aided Design (CAD) systems and Computer Aided Geometric Design (CAGD) [1-7]. NURBS is also promulgated by ISO to be the unique mathematical method for defining industrial product shapes in STEP.

The machining of NURBS complex surface is a research hotspot in the field of CNC community [8-12]. Additionally, robot machining is another important application field of NURBS complex curves and surfaces[13-16]. With the emergence of additive manufacturing (AM) technology, arc AM has made great progress in the past two decades[17-25]. However, the research of arc AM focuses on the contour wall and block shape composed of straight line and arc. The arc cladding preparation technology for complex surface protective coating has not attracted academic attention. Combining serial robot, complex surface and arc cladding to solve the arc cladding problem of NURBS complex surface protective coating based on serial robot platform is not only a work of great industrial practical application 
value, but also an urgent technology in the arc cladding industry of complex surface coating.

In the arc cladding process, plasma powder cladding has been widely used in industry because of its high arc column temperature, good arc column bunching (similar to the laser and electron beam), small heat affected zone, easy powder matching and low-cost [17-25]. It is also an important process for academic research on low-cost coating preparation.

The problem of cladding trajectory planning must be solved before plasma cladding complex surface coating. Trajectory planning is an important topic in the field of robotics. Different scholars have proposed different trajectory planning methods and achieved good results[26-28]. However, the trajectory planning of plasma cladding complex surface coating based on serial robot has not attracted scholars' attention. In order to solve the urgent needs of the industry and promote the development of the plasma cladding process, the trajectory planning of plasma powder cladding complex surface protective coating based on serial robot is researched in this work through proposing the concept of the NURBS function open/colosed-loop mapping.

\section{NURBS curves representation}

\subsection{NURBS curve representation}

A NURBS curve of $p$-degree is represented parametrically as [29]:

$$
\boldsymbol{C}(u)=\frac{\sum_{i=0}^{n} N_{i, p}(u) w_{i} \boldsymbol{P}_{i}}{\sum_{i=0}^{n} N_{i, p}(u) w_{i}} \quad(0 \leq u \leq 1)
$$

Where

$\boldsymbol{P}_{i}(i=0 \rightarrow n):$ NURBS curves control vertices, connected sequentially into control polygons;

$w_{i}(i=0 \rightarrow n)$ : the weights of $\boldsymbol{P}_{i} ;$

$p:$ degree of the NURBS curve;

$u:$ knot value $(\boldsymbol{u} \in \boldsymbol{U})$;

$\boldsymbol{U}$ : knot vector as:

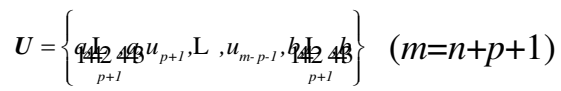

$N_{i, k}(u)(i=0 \rightarrow n)$ : the $p$ th order basis function. Recursive formulae for the basis function $N_{i, p}(u)$ can be expressed as:

$$
\left\{\begin{array}{l}
N_{i, 0}(u)= \begin{cases}1 & \text { for } \quad u_{i} \leq u<u_{i+1} \\
0 & \text { otherwise }\end{cases} \\
N_{i, k}(u)=\frac{u-u_{i}}{u_{i+k}-u_{i}} N_{i, k-1}(u)+\frac{u_{i+1}-u}{u_{i+k+1}-u_{i+1}} N_{i+1, k-1}(u) \\
\text { defined } \frac{0}{0}=0
\end{array}\right.
$$

Additionally, a rational basis function $R_{i, p}(u)$ can be defined as follows:

$$
R_{i, p}=\frac{N_{i, p}(u) \boldsymbol{w}_{i}}{\sum_{j=0}^{n} N_{j, p}(u) \boldsymbol{w}_{j}} \quad(0 \leq u \leq 1
$$

therefore, Eq.(1) can be expressed as:

$$
\boldsymbol{C}(u)=\sum_{i=0}^{n} R_{i, p}(u) w_{i} \boldsymbol{P}_{i} \quad(0 \leq u \leq 1
$$

\subsection{NURBS surface representation}

A NURBS surface is a bivariate vector-valued piecewise rational function. The NURBS surface is expressed as follows:

$$
\boldsymbol{S}(u, v)=\frac{\sum_{i=0}^{n} \sum_{j=0}^{m} N_{i, p}(u) N_{j, q}(v) w_{i, j} \boldsymbol{P}_{i, j}}{\sum_{i=0}^{n} \sum_{j=0}^{m} N_{i, p}(u) N_{j, q}(v) w_{i, j}} \quad(0 \leq u, v \leq 1)
$$

Where

$\boldsymbol{P}_{i, j}$ : forming a bidirectional control point net;

$\boldsymbol{\omega}_{i, j}$ : the weights;

$N_{i, p}(u)$ and $N_{j, q}(u)$ : the nonrational B-spline basis functions defined on $\boldsymbol{U}$ and $\boldsymbol{V}$, respectively.

The knot vectors of $\boldsymbol{U}$ and $\boldsymbol{V}$ are presented as:

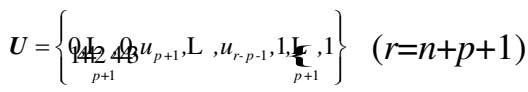

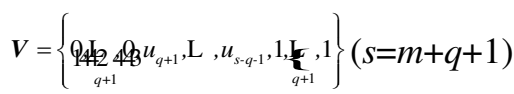

Furthermore, the piecewise rational basis function of $\left\{R_{i, j}(u, v)\right\}$ can be defined as follows:

$$
R_{i, j}(u, v)=\frac{N_{i, p}(u) N_{j, q}(v) w_{i, j}}{\sum_{k=0}^{n} \sum_{l=0}^{m} N_{k, p}(u) N_{l, q}(v) w_{k, l}} \quad(0 \leq u, v \leq 1)
$$

As a result, Eq.(6) is rewritten as:

$$
\boldsymbol{S}(u, v)=\sum_{i=0}^{n} \sum_{j=0}^{m} R_{i, j}(u, v) \boldsymbol{P}_{i, j}
$$

\section{Robotic plasma cladding of NURBS curve coating}

A plasma - computer integrated cladding is constructed by integrating a Motoman-UP6 serial robot with a DML-V02BD plasma power (Shanghai Duomu) as shown in Fig.1a. The robotic kinematic 
calculation is carried out using the $\mathrm{D}-\mathrm{H}$ convention [30-32], and the D-H parameters of the Motoman-UP6 is demostrated in Fig.1b. is based on the cladding of complex curve. The relationship between a robot and a NURBS curve is shown in Fig.2.

The plasma cladding of complex surface coating

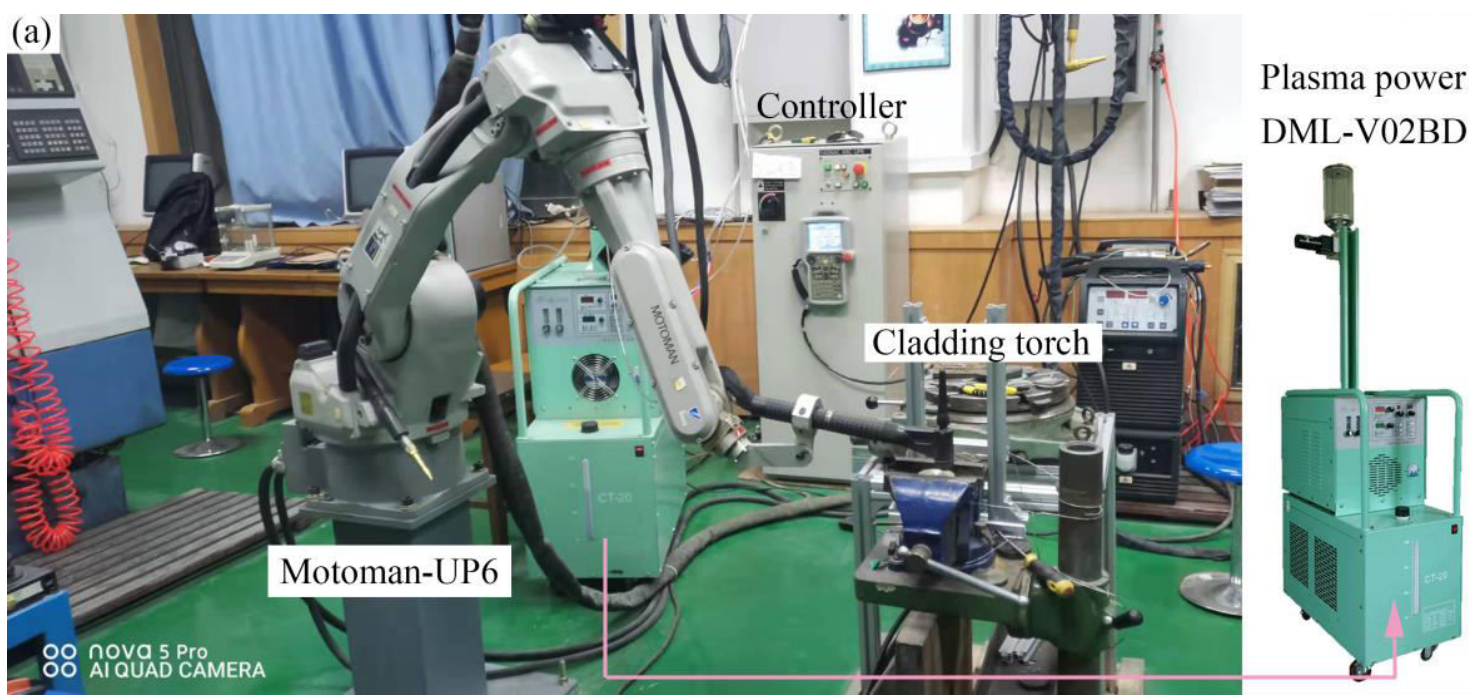

(b)
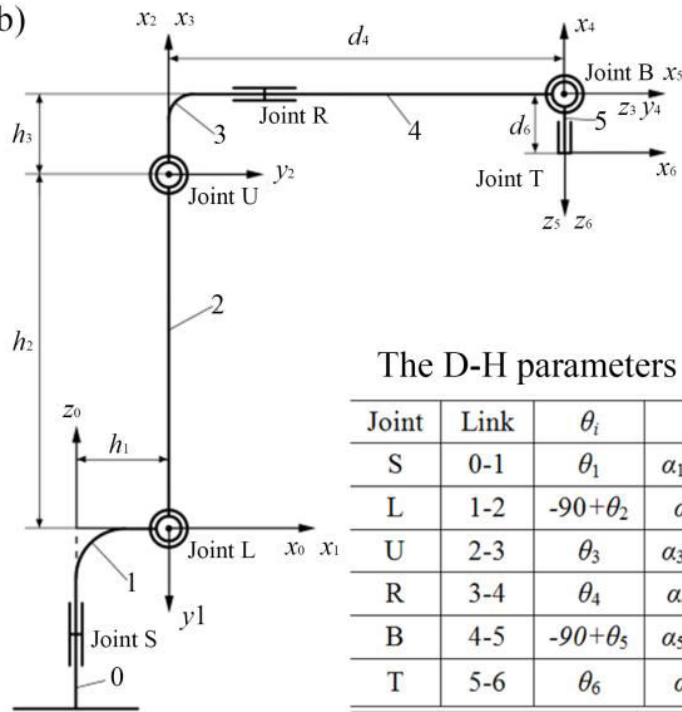

The D-H parameters of every link for Motoman UP6

\begin{tabular}{c|c|c|c|c|c|c}
\hline Joint & Link & $\theta_{i}$ & $a_{i}$ & $a_{i}$ & $d_{i}$ & range \\
\hline $\mathrm{S}$ & $0-1$ & $\theta_{1}$ & $\alpha_{1}=-90$ & $a_{1}=150$ & $d_{1}=0$ & {$[-170+170]$} \\
\hline $\mathrm{L}$ & $1-2$ & $-90+\theta_{2}$ & $\alpha_{2}=0$ & $a_{2}=570$ & $d_{2}=0$ & {$[-90+150]$} \\
\hline $\mathrm{U}$ & $2-3$ & $\theta_{3}$ & $\alpha_{3}=-90$ & $a_{3}=130$ & $d_{3}=0$ & {$[-170+190]$} \\
\hline $\mathrm{R}$ & $3-4$ & $\theta_{4}$ & $\alpha_{4}=90$ & $a_{4}=0$ & $d_{4}=640$ & {$[-180+180]$} \\
\hline $\mathrm{B}$ & $4-5$ & $-90+\theta_{5}$ & $a_{5}=-90$ & $a_{5}=0$ & $d_{5}=0$ & {$[-225+45]$} \\
\hline $\mathrm{T}$ & $5-6$ & $\theta_{6}$ & $\alpha_{6}=0$ & $a_{6}=0$ & $d_{6}=95$ & {$[-360+360]$} \\
\hline
\end{tabular}

Fig.1 Motoman-UP6 robot linkage coordinate systems based on the D-H convention. a The plasma - computer integrated cladding system. b The D-H parameters of the linkage coordinate systems [32].

The NURBS curve interpolation has to be implemented before cladding a NURBS curve. Actually, the NURBS curve plasma cladding is a process in which the robot controls the cladding torch to run along the fitting straight line segments between interpolation points from $\boldsymbol{C}\left(u_{i-1}\right)$ to $\boldsymbol{C}\left(u_{i+n}\right)$ as shown in Fig.2a. The NURBS curve interpolation is the process of finding the coordinates of interpolation point $\boldsymbol{C}\left(u_{i}\right)$. Then, the point $\boldsymbol{C}\left(u_{i}\right)$ is used as the position point in the homogeneous transformation matrix $\boldsymbol{T}$ of the cladding torch control point. The posture $[\boldsymbol{n} \boldsymbol{o} \boldsymbol{a}]$ of the cladding torch is determined by process requirements.
Furthermore, the robotic joint coordinates $\boldsymbol{\theta}$ is calculated according to matrix $\boldsymbol{T}$ using serial robotic inverse kinematics[30, 32].

A NURBS curve like Pikachu as shown in Fig.2b is designed for the research of the plasma cladding of NURBS surface coating in this work. Its parameters are outlined in Appendix A.

The interpolation has always been an important problem in NURBS curve research, which is related to the machining accuracy and efficiency of $\mathrm{CNC}$ machine tools. In our previous research [33], the equi-arc length interpolation with the fixed step plus 
golden section search (EALI-FS+GSS) is proposed. This interpolation method can realise the uniform distribution of interpolation points with the equi-arc

(a)

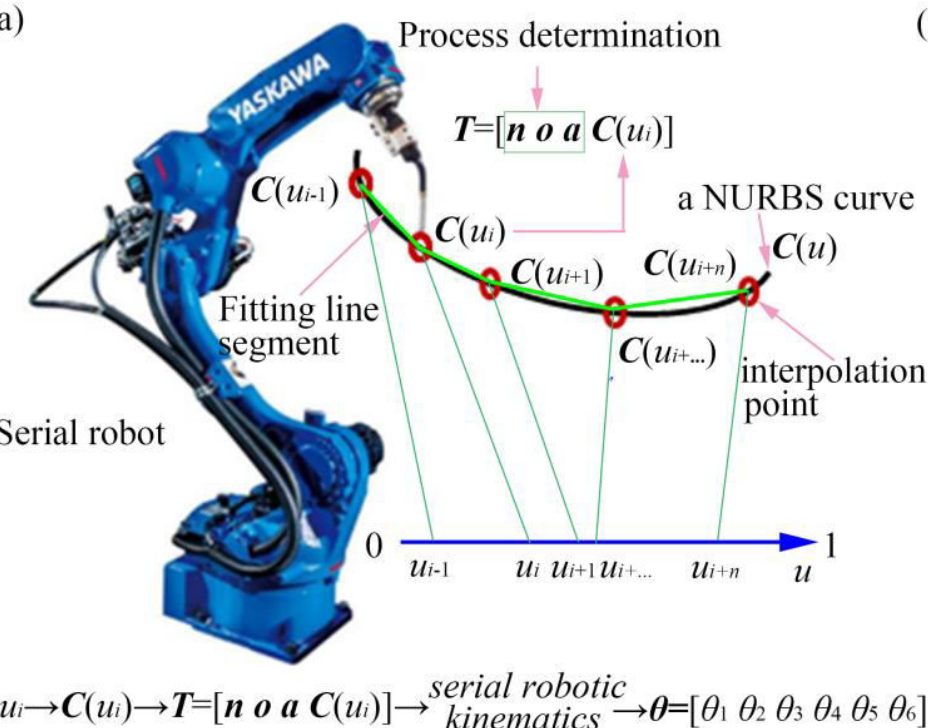

length, and used in this work to research the plasma cladding of the NURBS surface coating.

(b)

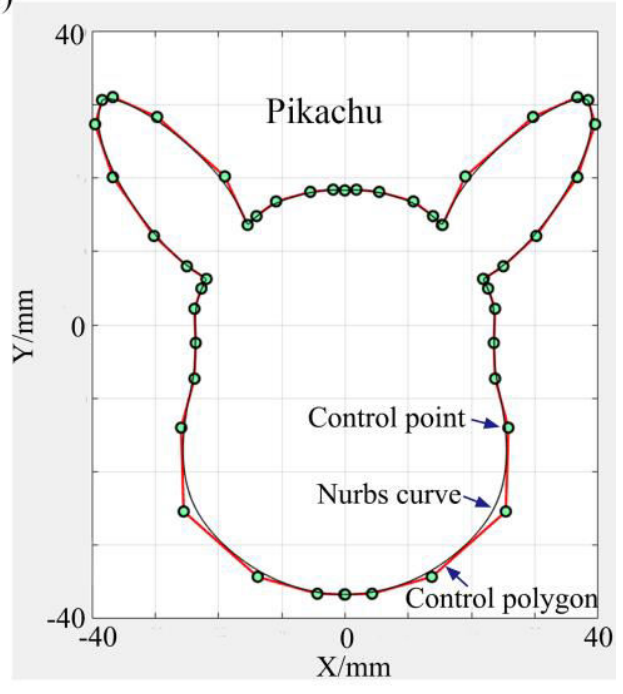

Fig.2 Robotic plasma cladding of NURBS curve coating. a Robot and NURBS curve. b the NURBS curve of Pikachu.

\section{NURBS function closed-loop mapping trajectory planning}

\subsection{Principle of NURBS function open/ closed-loop mapping}

The NURBS function open/closed-loop mapping curve is proposed to solve the problem of the plasma cladding trajectory planning for complex NURBS surface based on the serial robot of Motoman UP6 as shown in Fig.1a.

The NURBS function mapping (NURBS FM) is the mapping process from $u$ to $S(u, v)$ as shown in Fig.3. When $u_{i}$ is taken from 0 to 1 on the $u$ axis, it can be mapped into a NURBS curve $\boldsymbol{C}(u)$ using Eq.(1). To facilitate trajectory planning and design, this NURBS curve can be define to be a 2D-NURBS curve as shown in Fig.3. Thus, the $2 \mathrm{D}$ space where the NURBS curve $\boldsymbol{C}(u)$ is located can be used as the design space of trajectory planning. The coordinate $(x, y)$ of $\boldsymbol{C}(u)$ in Cartesian coordinates can be used as variables of $\boldsymbol{S}(u, v)$ via Eq.(6). Consequently, the $\boldsymbol{C}\left(u_{i}\right)$ point on the planar NURBS curve $\boldsymbol{C}(u)$ is mapped into the $\boldsymbol{S}\left(u_{i}, v_{i}\right)$ point on the NURBS surface $\boldsymbol{S}(u, v)$. All $\boldsymbol{S}\left(u_{i}, v_{i}\right)$ points on the surface form a 3D spacial curve called NURBS FM curve. It should be pointed out that this 3D spacial curve is not a NURBS curve. In plasma cladding, the 2D-NURBS curve is the planning curve on the design plane, and the NURBS FM curve is the plasma cladding trajectory on the complex NURBS surface. Fig.4a-d take Pikachu as an example to clearly express the relationship between the 2D-NURBS curve and the NURBS FM curve.

It can be known from the above NURBS FM curve principle:

(1) $u$ is the $1 \mathrm{D}$ variable on the $u$ linear axis.

(2) $\boldsymbol{C}(u)$ is the 2D-NURBS curve of the plasma cladding trajectory planning.

(3) The NURBS FM curve is a 3D spacial curve on the NURBS surface $\boldsymbol{S}(u, v)$.

Depending on where the constraint is applied, NURBS FM can be divided into open-loop and closed-loop. The constraint refers to the properties between interpolation points using the fixed step (FS) interpolation, the equal chord length (ECL) interpolation, the equal arc length (EAL) interpolation and the equal bow height (EBH) interpolation, etc.

NURBS function open-loop mapping (NURBS-FOLM) is carried out along the route of $u \rightarrow \boldsymbol{C}(u) \rightarrow \boldsymbol{S}(u, v)$ as shown in Fig.3. By applying the FS/ECL/EAL/EBH constraint on the 2D plane, the NURBS FM curve on the given $\boldsymbol{S}(u, v)$ NURBS surface from the constrained planar NURBS curve $C(u)$ can be obtained, which is the NURBS-FOLM. 
The spacial curve on the given NURBS surface $S(u, v)$ is defined as NURBS-FOLM curve. Obviously, the FS/ECL/EAL/EBH constraint is only valid for the 2D-NURBS curve $\boldsymbol{C}(u)$ and not for the spacial NURBS-FOLM curve in NURBS-FOLM.

NURBS function closed-loop mapping (NURBS-FCLM) is carried out along the route of (1) $\rightarrow$ (2) $\rightarrow$ (3) $\rightarrow$ (4) $\rightarrow$ (1) $\rightarrow$ as shown in Fig.3. The ECL/EAL/EBH constraint is applied on the NURBS surface $\boldsymbol{S}(u, v)$. The (1) $\rightarrow$ (2) $\rightarrow$ (3) $\rightarrow$ (4) $\rightarrow$ (1) $\rightarrow$ route calculation is repeated until the error $e$ is met. This is NURBS-FCLM. The spacial curve on the given
NURBS surface $S(u, v)$ is defined as NURBS-FCLM curve. Contrary to NURBS-FOLM, the ECL/EAL/EBH constraint is only valid for the NURBS FM curve on the NURBS surface $S(u, v)$ and not for the 2D-NURBS curve $\boldsymbol{C}(u)$.

Both NURBS-FOLM and NURBS-FCLM obtain the plasma cladding trajectory on the predefined NURBS surface to accomplish the cladding trajectory planning. Fig.4e and $\mathrm{f}$ show the the correspondence between interpolation points and mapping points on the 2D-NURBS curve and the NURBS FM curve, respectively.

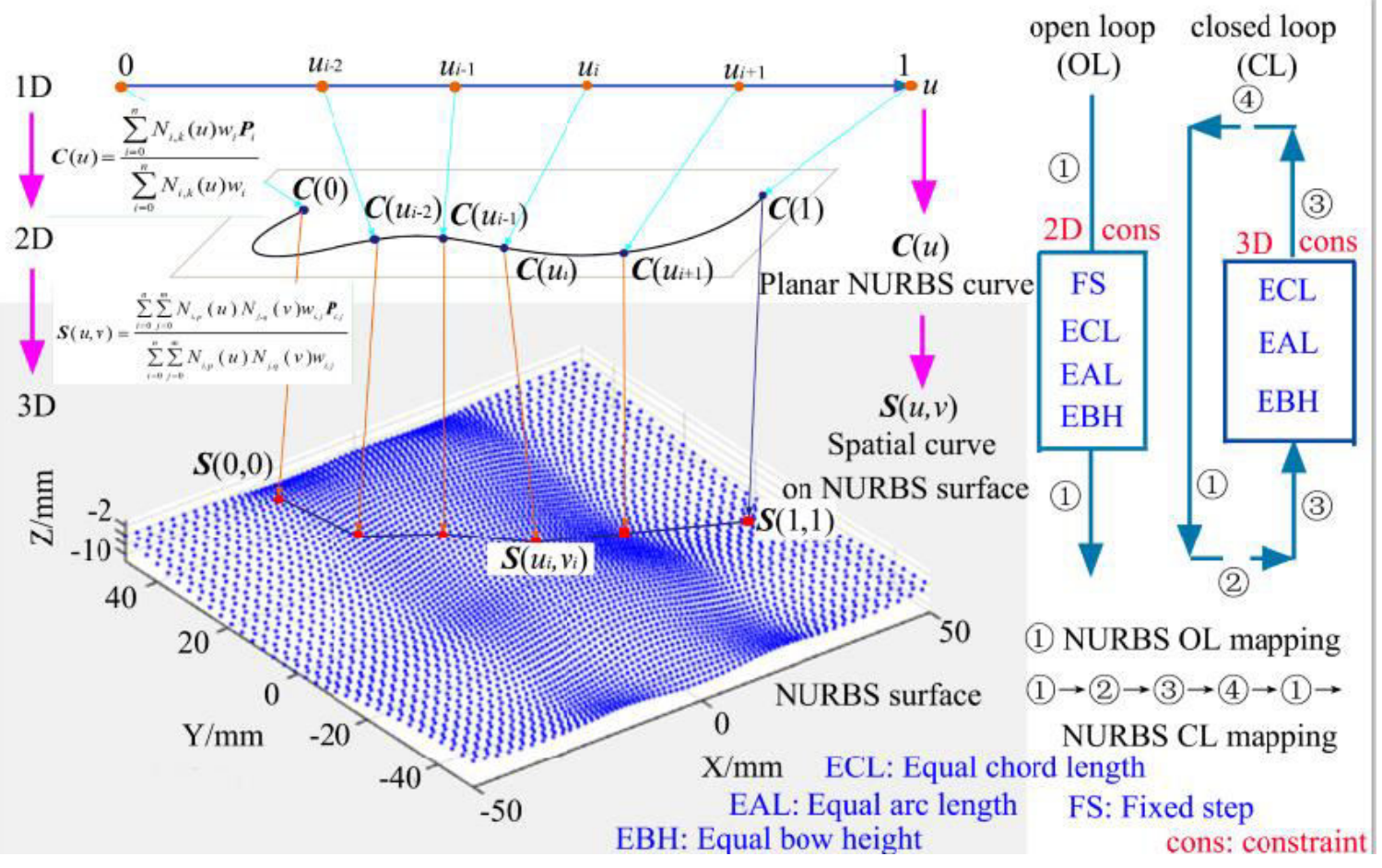

Fig.3 The schematic of the NURBS function open/closed mapping.

(a)

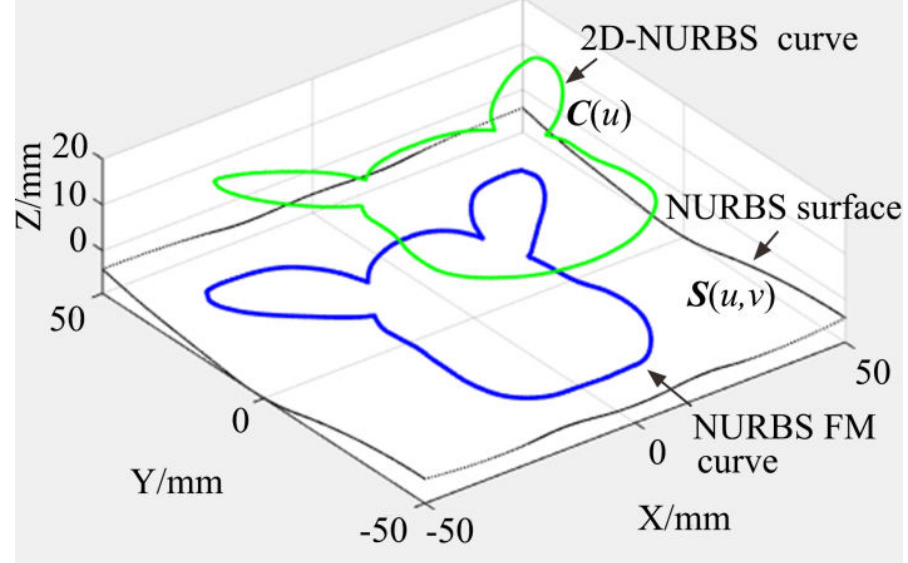

(b)

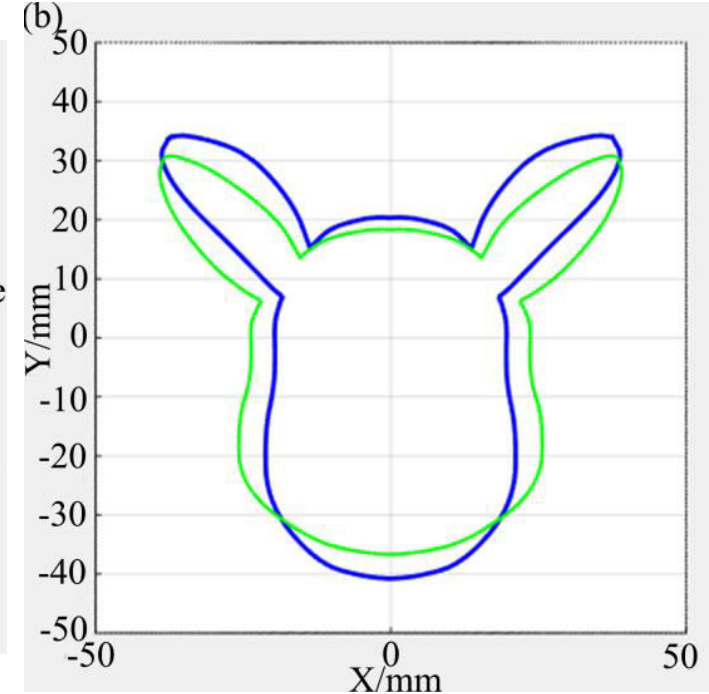




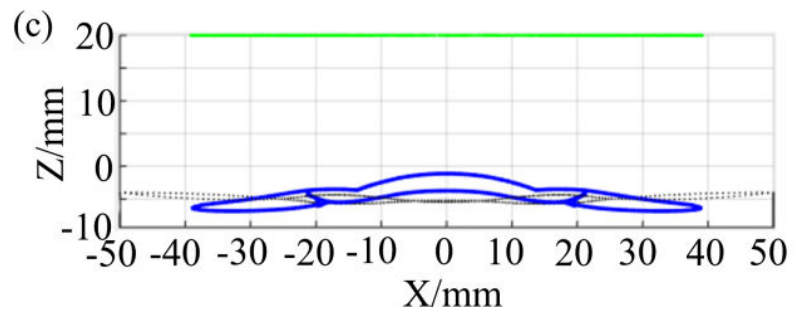

(e)

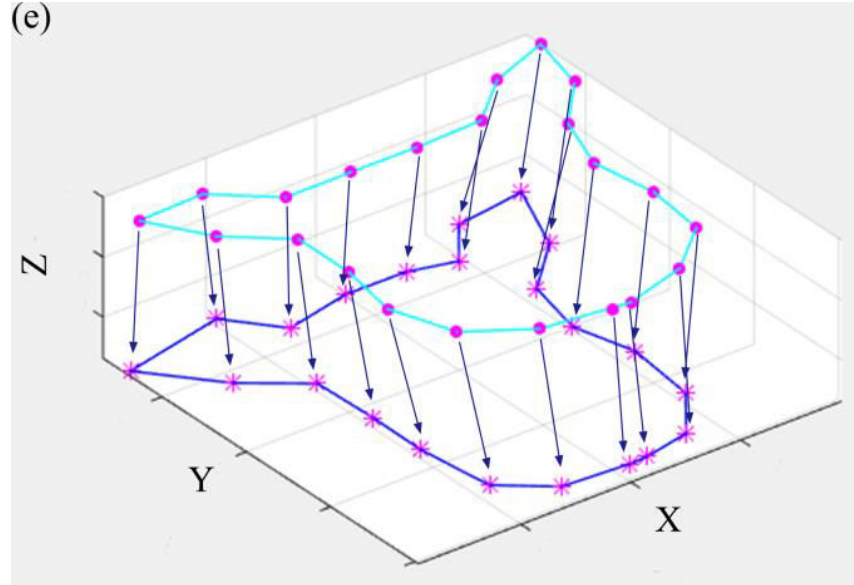

(d)

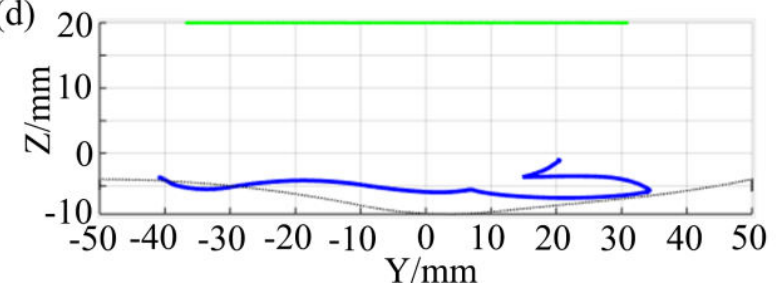

(f)

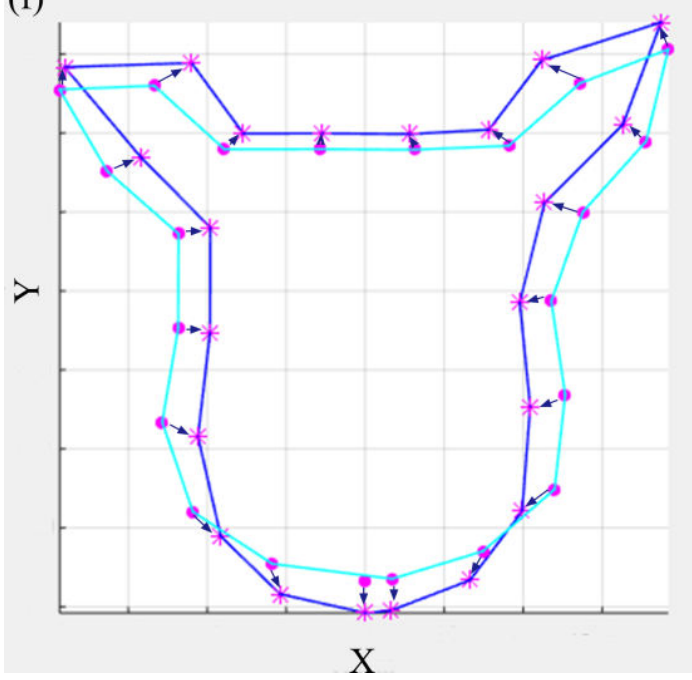

Fig.4 The NURBS function mapping of the Pikachu curve. $\mathbf{a} \sim \mathbf{d} 2 \mathrm{D}$ NURBS curve and NURBS function mapping curve in 3D-view, 2D-view, XZ-view and YZ-view, respectively. e and f The schematic of the correspondence between interpolation points and mapping points in 3D-view and 2D-view, respectively.

Fig.5 demonstrates the NURBS-FOLM and NURBS-FCLM in block digram. The NURBS FM is the mapping relationship between three spaces. The first space is the $1 \mathrm{D}$-space containing the $u$-axis, the second space is the 2D-space containing the planar NURBS curve $\boldsymbol{C}(u)$, and the third space is the 3D-space containing the NURBS surface $S(u, v)$. The position where the constraint is applied is clearly shown in Fig.5. Taking the EAL constraint as an example, the NURBS-FOLM means that the arc length between interpolation points on the NURBS curve $\boldsymbol{C}(u)$ is equal, while the NURBS-FCLM means that the arc length between mapping points on the
NURBS surface $S(u, v)$ is equal. For this reason, the search cycle path implemented to meet the constraint is also different. For the NURBS-FOLM, the search cycle path is located between $u$ and $\boldsymbol{C}(u)$ to meet the condition of $\left|s_{i}-s\right| \leqslant e\left(s_{i}\right.$ and $s$ are the calculated arc length and the designated arc length of 2D-NURBS curve $\boldsymbol{C}(u)$ respectively, $e$ is error ). Contrarily, For the NURBS-FCLM, the search cycle path is located between $u$ and the NURBS-FM curve to meet the condition of $\left|S_{i}-S\right| \leqslant e\left(S_{i}\right.$ and $S$ are the calculated arc length and the designated arc length of NURBS-FM curve respectively, $e$ is error ) as shown in Fig.5.

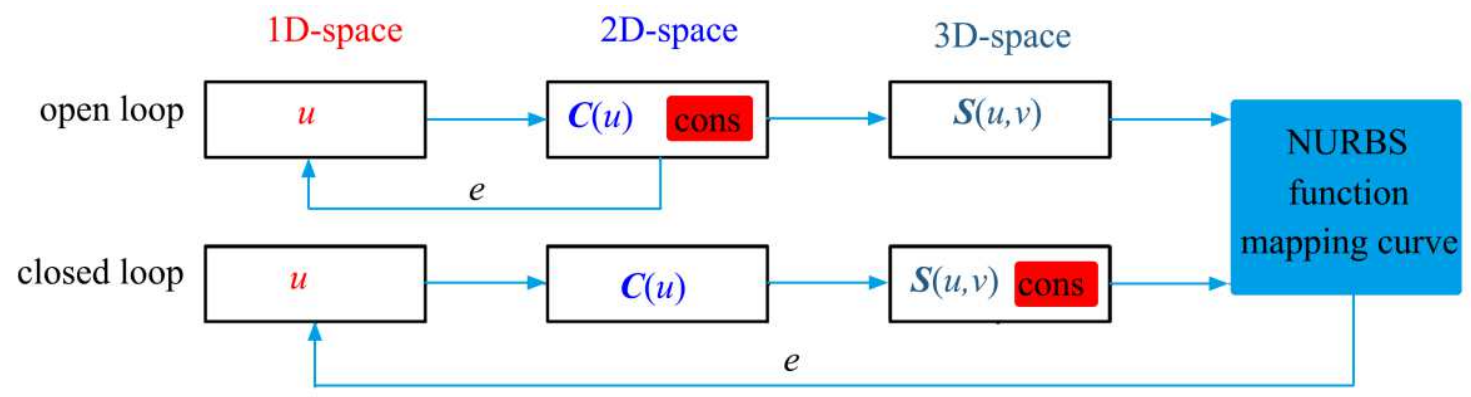

Fig.5 The block diagram of the NURBS function open/closed mapping. 


\subsection{Simulation of NURBS function open/closed}

The EAL constraint used the EALI-FS+GSS method is employed in this work to [33]. The distribution of interpolation points on the 2D-NURBS curve and the NURBS-FM curve using

(a)

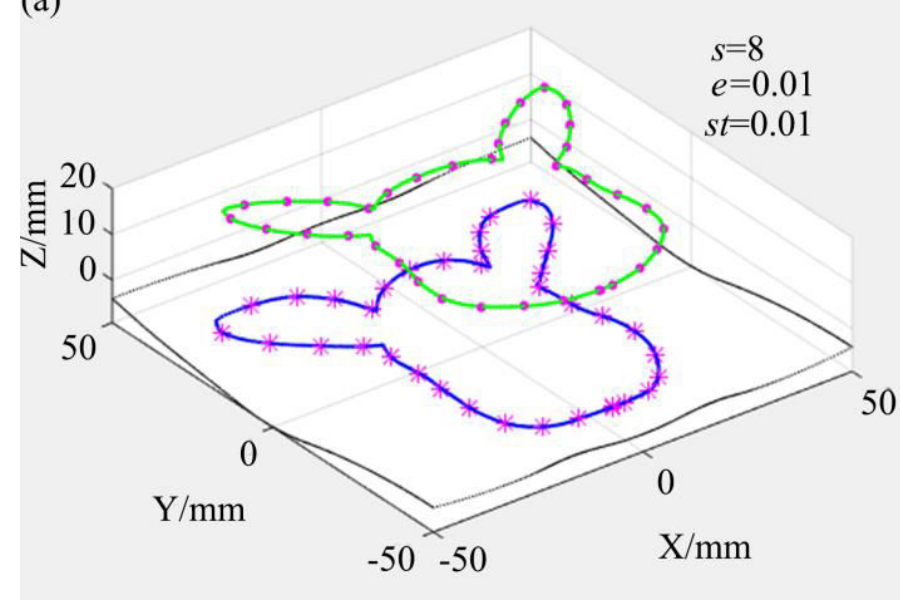

(c)

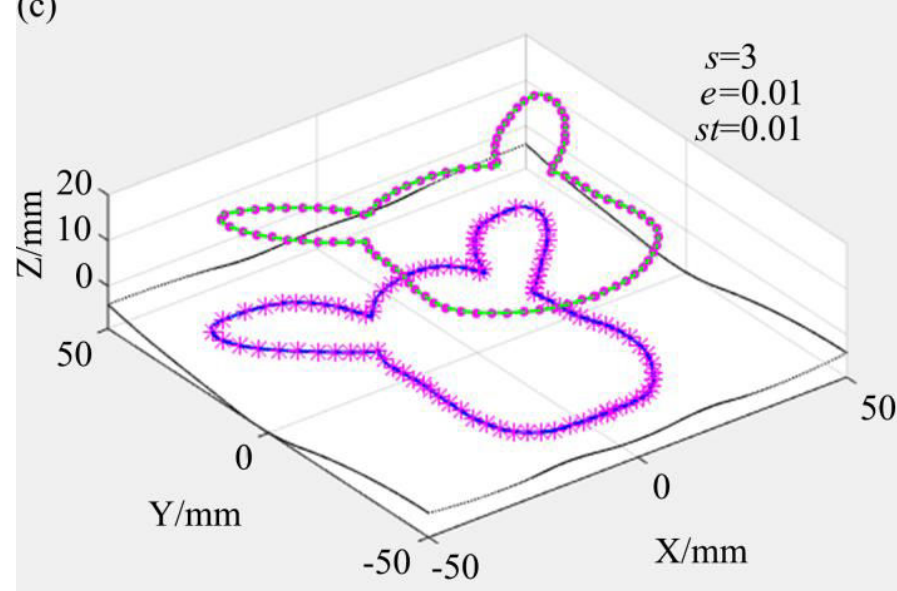

(e)

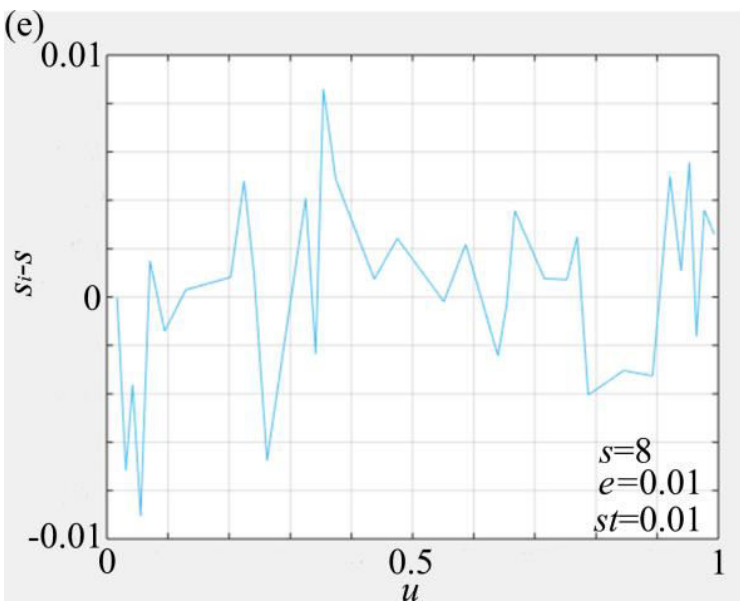

\section{-loop mapping}

NURBS-FOLM with different parameters is shown in Fig.6a-d. Fig.6e and $\mathrm{f}$ show that the distribution of interpolation points on 2D-NURBS curve meet the constraint of EAL constraint.

(b)

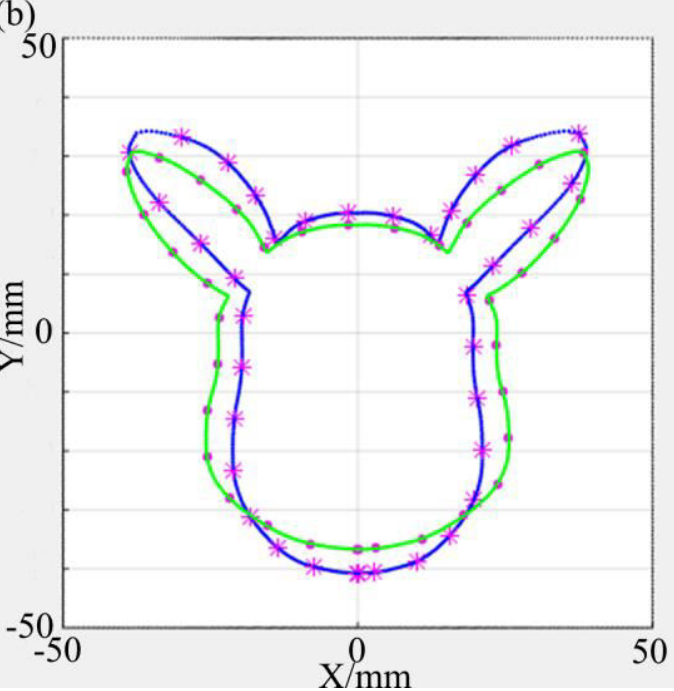

(d)
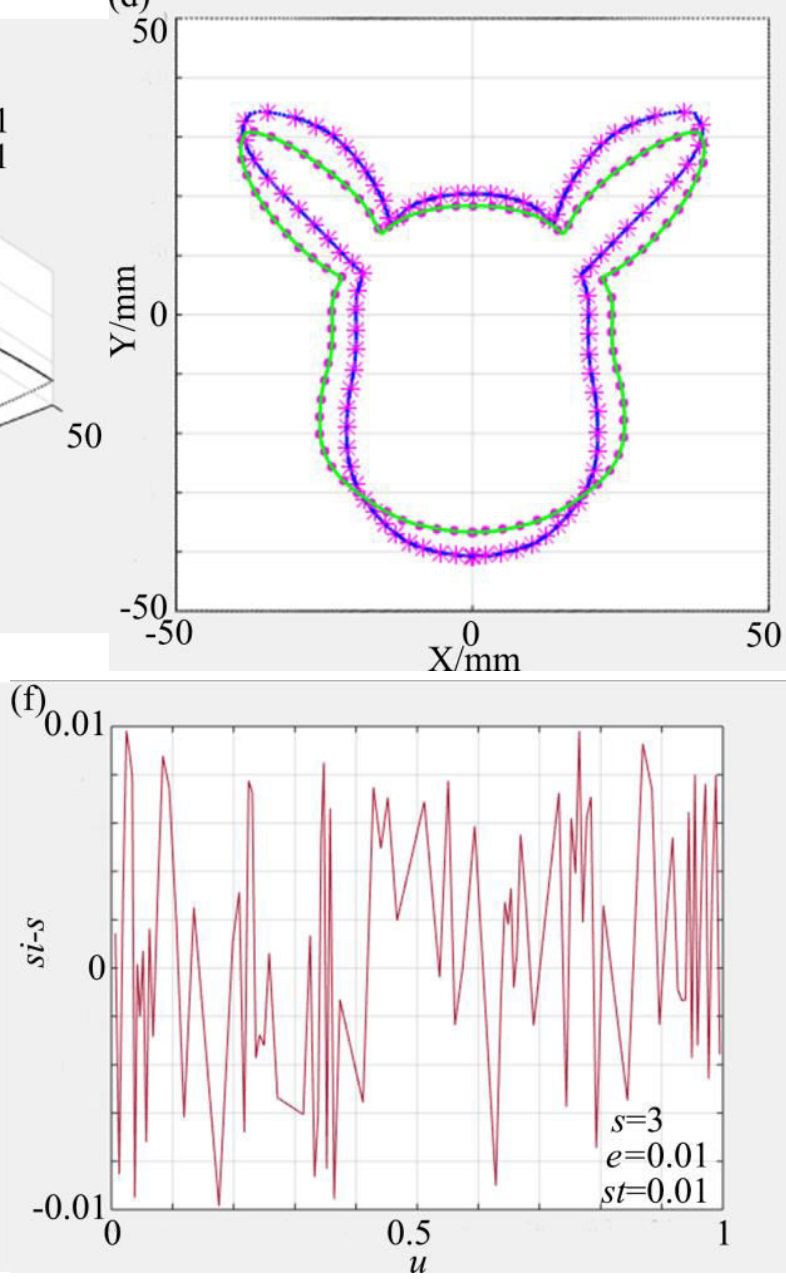

Fig.6 The NURBS function open-loop mapping of the Pikachu curve. a and $\mathbf{b} 3 \mathrm{D}$ and 2D-view, respectively, [ $s e$ $s t]=\left[\begin{array}{lll}8 & 0.01 & 0.01\end{array}\right] . \mathbf{c}$ and $\mathbf{d}$ 3D and 2D-view, respectively, [s e $\left.s t\right]=\left[\begin{array}{llll}3 & 0.01 & 0.01\end{array}\right] . \mathbf{e}\left(s_{i}-s\right)$-curve, $\left[\begin{array}{lll}s & e & s t\end{array}\right]=\left[\begin{array}{ll}8 & 0.01\end{array}\right.$ 0.01]. f $\left(s_{i}-s\right)$-curve, $\quad\left[\begin{array}{ll}s & e\end{array} t\right]=\left[\begin{array}{llll}3 & 0.01 & 0.01\end{array}\right] .\left(s_{i}\right.$ and $s$ are calculated arc length and designated arc of 2D-NURBS curve $C(u)$, respectively, $e$ is error, $s t$ is the fixed step. ) 
Similarly, the distribution of interpolation points on the 2D-NURBS curve and the NURBS-FM curve using NURBS-FCLM with different parameters is

(a)

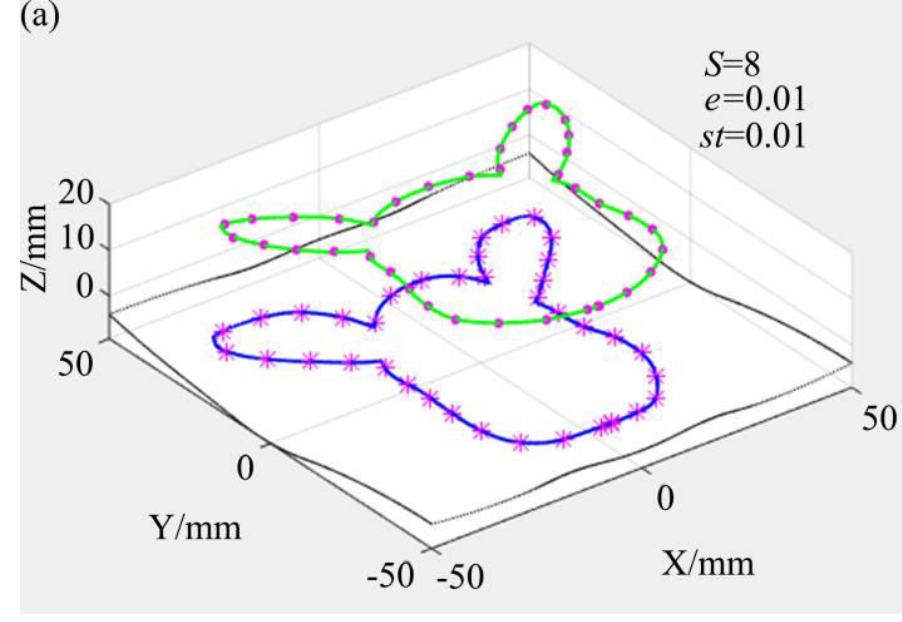

shown in Fig.7a-d. Fig.7e and $f$ show that the distribution of interpolation points on the NURBS-FM curve meet the constraint of EAL constraint.

(b)

(c)
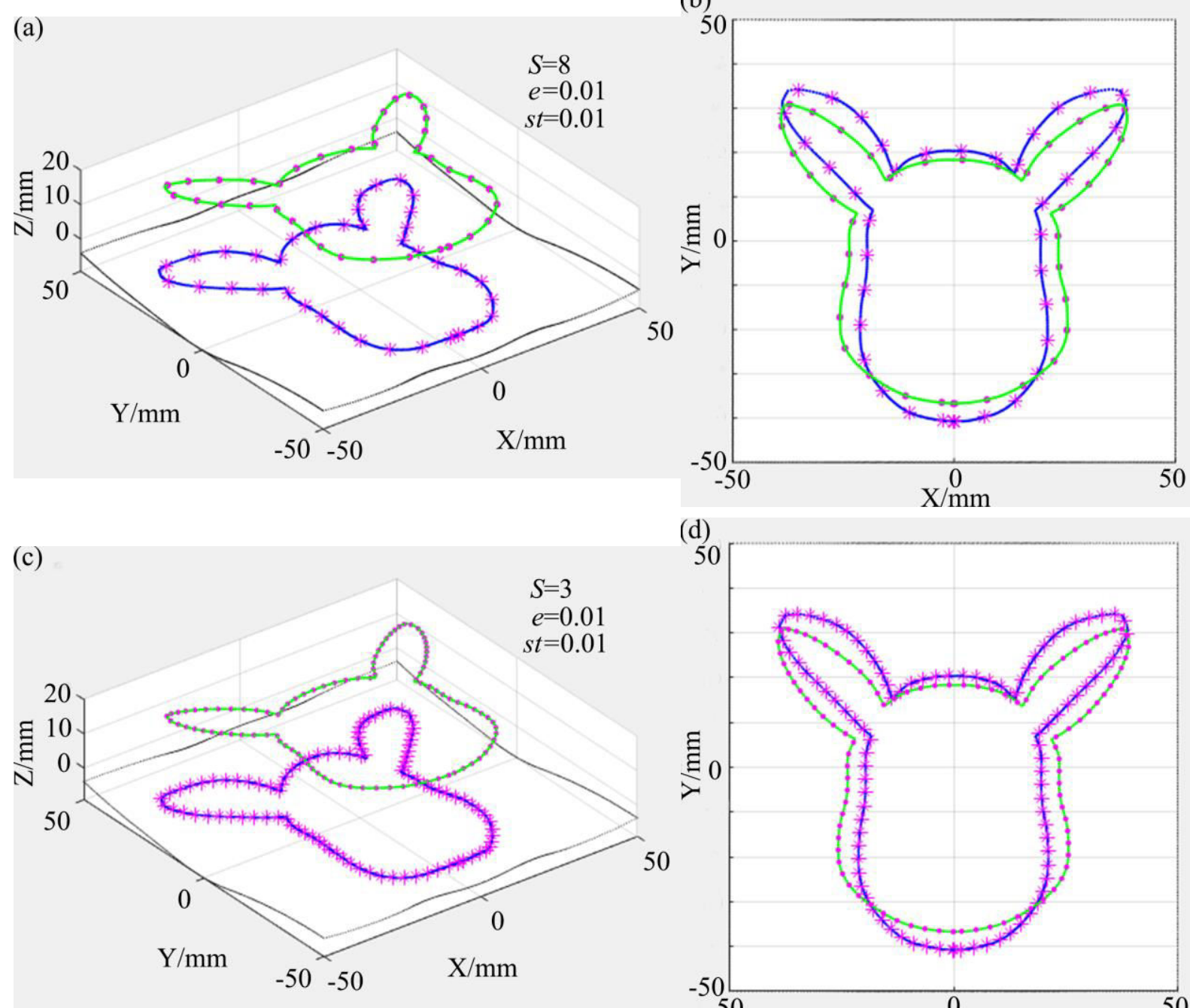

(d)

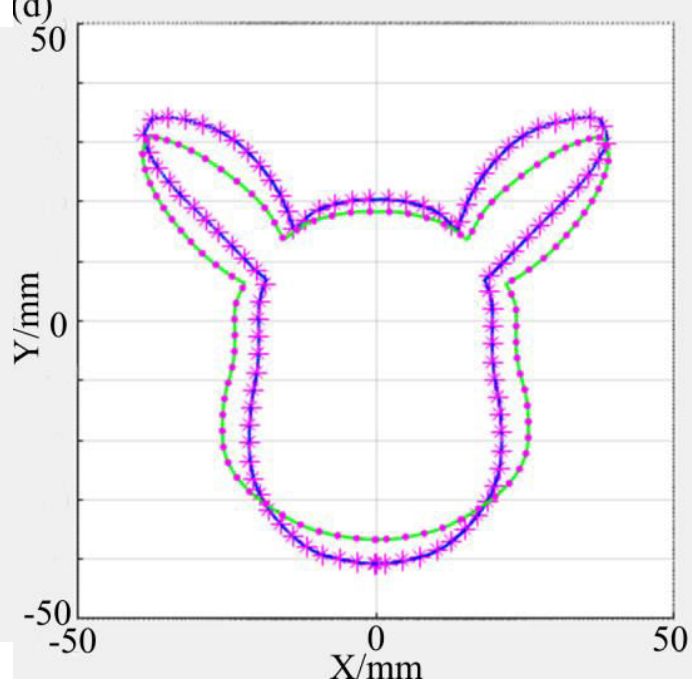

(e)

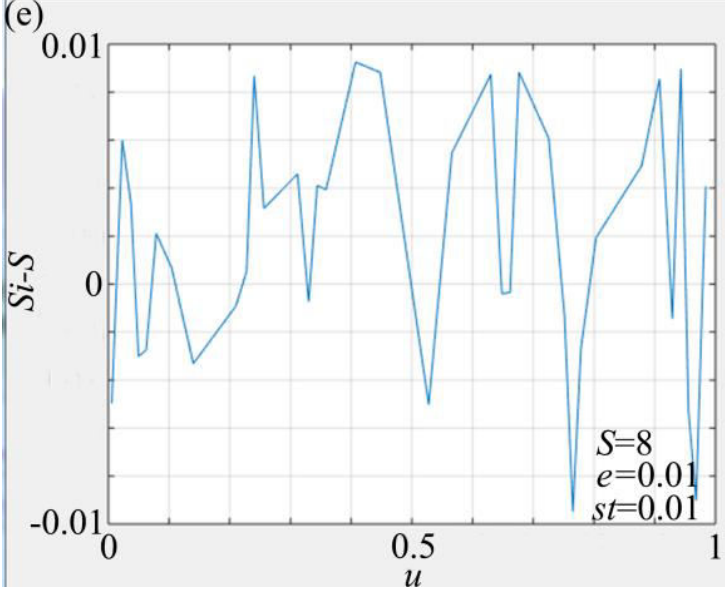

(f)

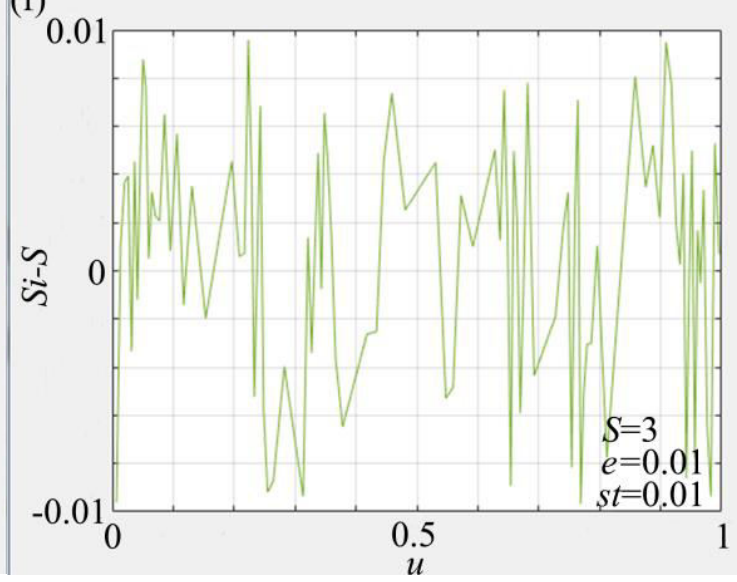

Fig.7 The NURBS function closed-loop mapping of the Pikachu curve. a and $\mathbf{b} 3 \mathrm{D}$ and 2D-view, respectively, [S

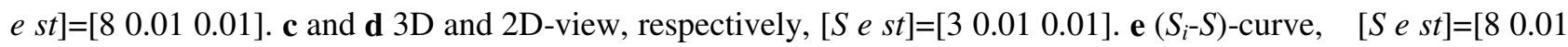
0.01]. f $\left(S_{i}-S\right)$-curve, $\quad\left[\begin{array}{ll}S & \text { e }\end{array} t\right]=\left[\begin{array}{lll}3 & 0.01 & 0.01\end{array}\right] .\left(S_{i}\right.$ and $S$ are calculated arc length and designated arc of NURBS-FM curve, respectively, $e$ is error, $s t$ is the fixed step. ) 
$s=S=8$ can be observed clearly in Fig.8. For example, the distribution of the interpolation points under the same EAL-value constraint in region A (Fig.8a) and region $\mathrm{B}$ (Fig.8b) are obviously different due to different constraint positions. Obviously, the uniform distribution of interpolation points on the NURBS-FM

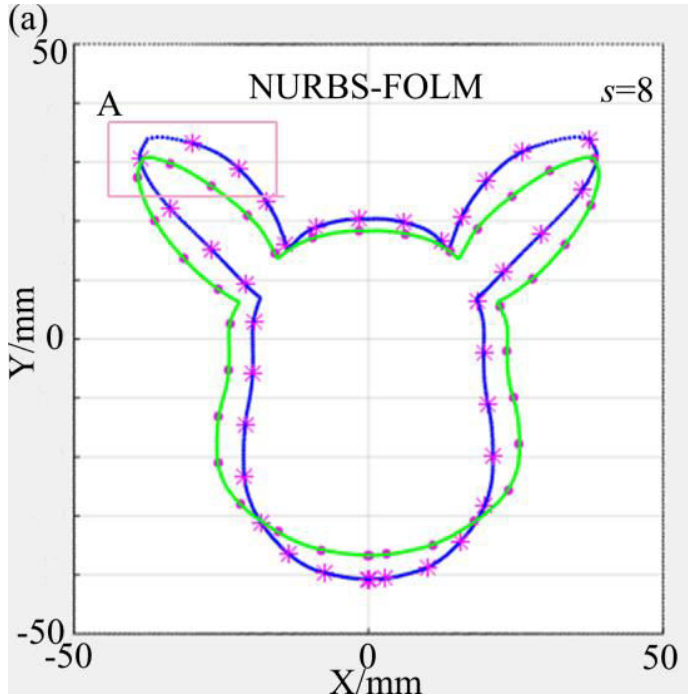

curve can be obtained by using NURBS- FCLM. Thus, the connected polygon (as shown in Fig.e and f) between interpolation points can approach the NURBS-FM curve with higher accuracy under smaller EAL-value constraint.

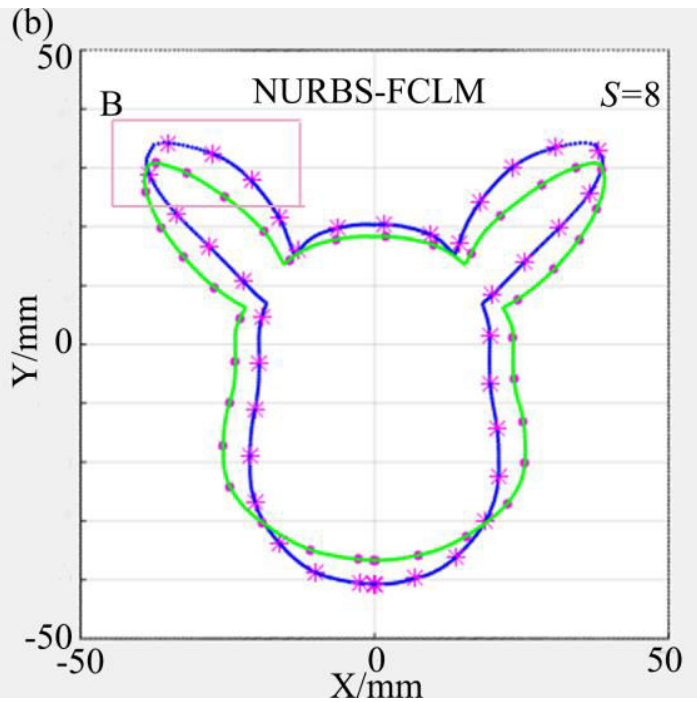

Fig.8 The distribution comparation of interpolation points between NURBS-FOLM and NURBS-FCLM. a NURBS-FOLM. b NURBS-FCLM.

\section{The plasma cladding of complex NURBS curve coating using NURBS-FCLM}

Before plasma cladding the NURBS-FM curve coating, the predefined surface should be machined based on a CNC milling machine equipped with FANUC $0 i$-MB. The pretreatment containing surface digital processing and milling is shown in Fig.9. The complex parameters in Appedix B are input into rhion 5 software, and processed the CAM in UG. After that, the complex surface workpiece is clamped on the plasma - computer integrated cladding system as show in Fig.10a.

A software is developed for the plasma cladding of complex NURBS surface coatings including the surface design function, trajectory planning function, serial robotic kinematic function and program generation function. Based on this software, the plasma cladding program for this cladding trajectory is generated.

The posture of the cladding torch relative to the workpiece as shown in Fig.10a. Q235 is used in this experiment as the substrate, while $\mathrm{AlCoCrCuNiNb}$ high entropy alloy powder is employed as the powder. The plasma cladding process parameters are listed as follows:

(1) Ar flow: $6 \mathrm{~L} / \mathrm{min}$;

(2) Ion gas flow: $1.7 \mathrm{~L} / \mathrm{min}$;

(3) Current: 115A;

(4) Powder feeding speed: $15 \mathrm{~mm} / \mathrm{s}$;

(5) The distance between the cladding torch tip and the workpiece: 7 9 mm;

(6) Cladding feedrate: $1.4 \mathrm{~mm} / \mathrm{s}$;

(7) Interpolation parameter: $[d s t]=[0.050 .01]$.

The plasma cladding process and results of the NURBS-FM curve using NURBS-FCLM trajectory planning approach is shown in Fig.10b-f. The NURBS-FM curve coating is uniform and well formed. 


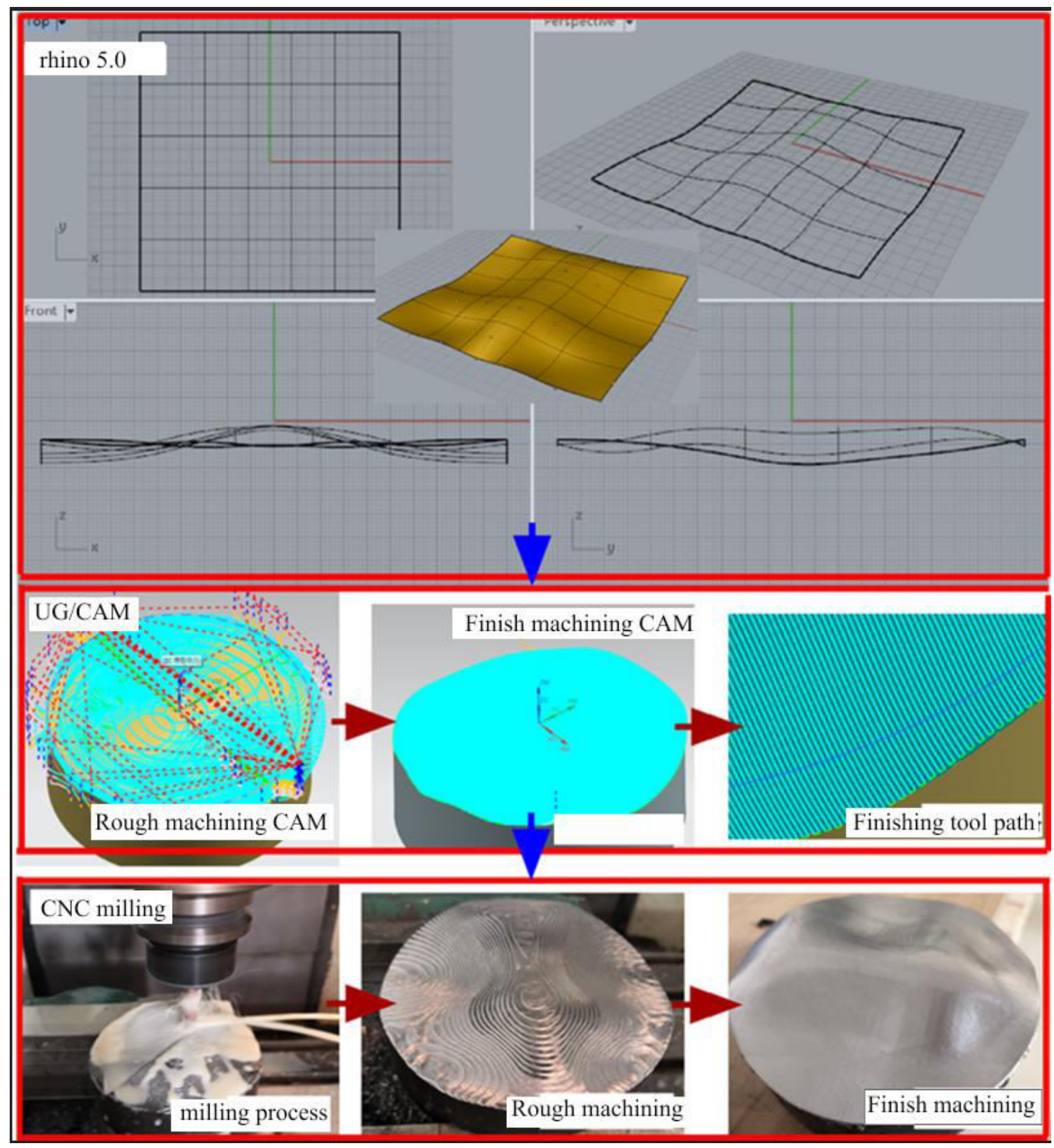

Fig.9 The pretreatment and milling of the NURBS surface.

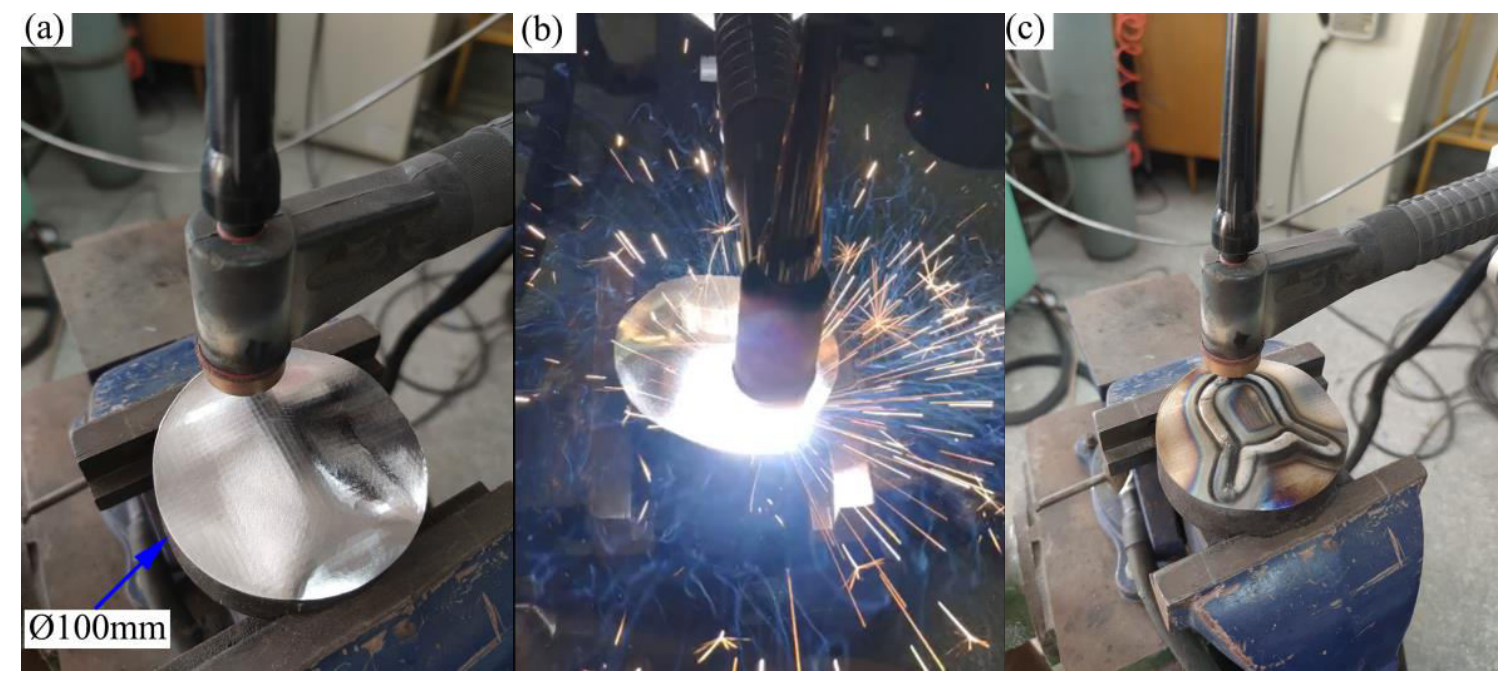




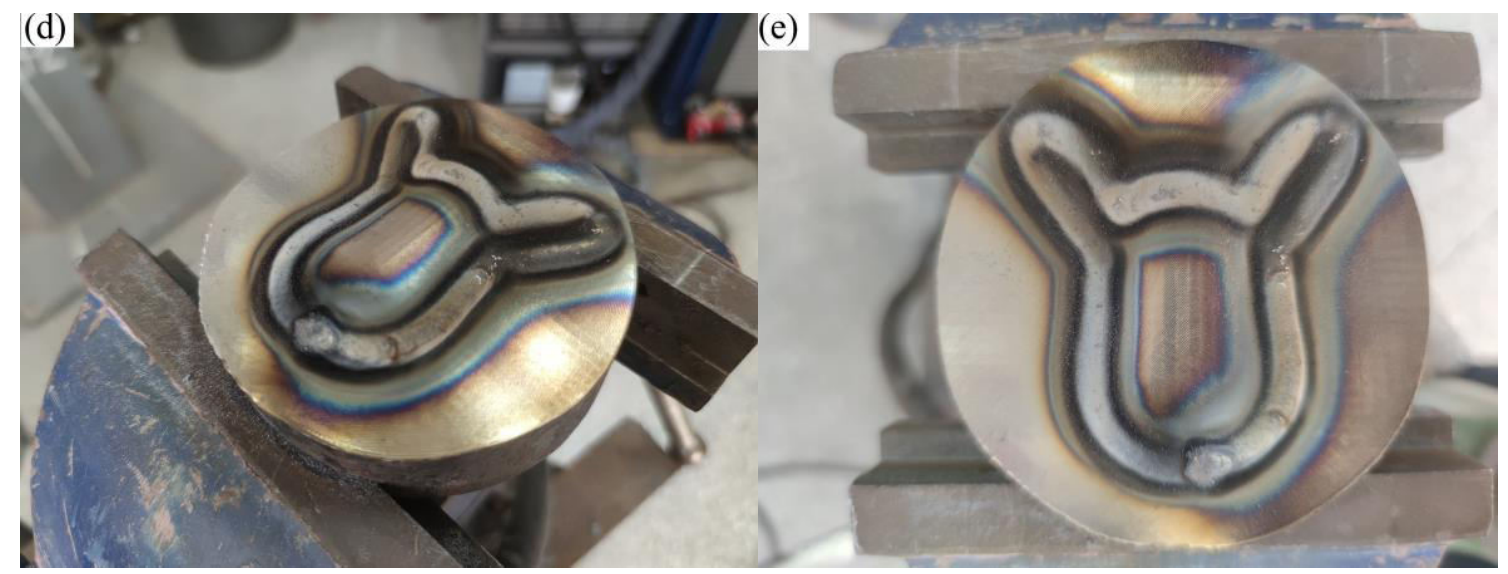

Fig.10 The plasma cladding process of the NURBS-FM curve using NURBS-FCLM trajectory planning approach. a before cladding. $\mathbf{b}$ cladding. $\mathbf{c}$ after cladding. d 3D-view. e XY-view.

\section{Conclusion/recommendation}

The trajectory planning is an important work for the complex surface manufacturing of computer integrated manufacuting system. It is a key task for the serial robotic plasma cladding of the NURBS surface coating based on a plasma - computer integrate cladding system. In order to solve the problem,

The concept of NURBS function open/closed -loop mapping is proposed in this work to explore the new approach of the trajectory planning of plasma cladding for comple NURBS surface coating.

The NURBS-FM curve is a $3 \mathrm{D}$ mapping curve (on a predefined NURBS surface) mapped from the 2D-NURBS curve using the NURBS surface function.

NURBS surface function mapping can be divided into open-loop mapping and closed-loop mapping according to the position applied by FS/ECL/EAL /EBH constraint.

The NURBS-FOLM is realized along the route of $u \rightarrow \boldsymbol{C}(u) \rightarrow \boldsymbol{S}(u, v)$, while the NURBS-FCLM along the route of $u \rightarrow \boldsymbol{C}(u) \rightarrow \boldsymbol{S}(u, v) \rightarrow u \rightarrow \ldots$. The constraint is applied to the 2D-NURBS curve in NURBS-FOLM, while to the 3D NURBS-FM curve in NURBS-FCLM. The NURBS-FOLM and NURBS-FCLM can ensure that the 2D-NURBS curve and 3D NURBS-FM curve has the ECL/EAL/EBH characteristics, respectively.

Regarding the NURBS-FM curve as plasma cladding trajectory, the NURBS-FCLM can provide engineers with a new ECL/EAL/EBH interpolation method for cladding trajectroy planning.

The simulation and experiment verifies the
NURBS-FCLM is feasible and effective. With the increasing application of surface protective coatings preparaed by plasma cladding, the NURBS-FCLM has broad application prospects inevitably.

\section{Declarations}

\section{- Funding}

This work was supported by National Natural Science Foundation of China (Grant numbers [52075235] and [51764038]), Employee Innovation Subsidy Fund Project of All China Federation of Trade Unions, Open Project of State Key Laboratory of Advanced Welding and Joining (Grant number [AWJ-21Z02]), Gansu Science and Technology Planning Project (Grant numbers [20YF8GA033], [17YF1GA018], [17CX1JA117], and [18JR3RA132]), Western Young Scholars of Chinese Academy of Sciences, Lanzhou Talent Innovation and Entrepreneurship Project (Grant numbers [2020-RC-120], [2019-RC-102], and [2018-RC-108]), Longyuan Youth Innovative and Entrepreneurial Talents Project, Foundation of A Hundred Youth Talents Training Program of Lanzhou Jiaotong University, and Gansu Provincial Employee Technology Innovation Subsidy Fund Project.

\section{- Conflicts of interest/Competing interests}

Not applicable.

\section{- Availability of data and material}

The data used to support the findings of this study are available from the corresponding author upon request. 


\section{- Code availability}

The research program code in this work is developed by the authors and is currently used for research and a small number of industrial applications.

\section{- Ethics approval}

Not applicable.

- Consent to participate

Not applicable.

- Consent for publication

Not applicable.

\section{- Authors' contributions}

All authors contributed to the study conception and design. Material preparation, data collection and analysis were performed by Zhaoqin Wang, Yu Shi, and Xiaorong Wang. The first draft of the manuscript was written by Zhaoqin Wang and all authors commented on previous versions of the manuscript. All authors read and approved the final manuscript.

\section{Acknowledgments}

We appreciated Yusen Wang, Ning Yang, Chengyu Li, Haonan Wei, Tong Yang, and Xu An for the softeware development.

\section{Appendix A:}

The design of a planar NURBS curve (like a Pikachu).

- The control points:

$$
\begin{aligned}
& \boldsymbol{P}=\{[0.01,-36.70,0.00] \quad[-4.38,-36.60,0.00] \\
& {[-13.80,-34.30,0.00] \quad[-25.50,-25.40,0.00]} \\
& {[-25.90,-14.00,0.00] \quad[-23.80,-7.32,0.00]} \\
& {[-23.60,-2.46,0.00] \quad[-23.80,2.19,0.00]} \\
& {[-22.70,4.94,0.00] \quad[-21.90,6.25,0.00]} \\
& {[-21.90,6.25,0.00] \quad[-25.00,7.97,0.00]} \\
& {[-30.20,12.10,0.00] \quad[-36.70,20.10,0.00]} \\
& {[-39.50,27.30,0.00] \quad[-38.40,30.60,0.00]} \\
& {[-38.40,30.60,0.00] \quad[-36.70,31.00,0.00]} \\
& {[-29.70,28.30,0.00] \quad[-19.00,20.20,0.00]} \\
& {[-15.40,13.60,0.00] \quad[-15.40,13.60,0.00]} \\
& {[-14.00,14.80,0.00] \quad[-10.90,16.80,0.00]} \\
& {[-5.49,18.10,0.00] \quad[-1.89,18.40,0.00]} \\
& {[-0.05,18.30,0.00] \quad[-0.05,18.30,0.00]} \\
& {[1.79,18.40,0.00] \quad[5.39,18.10,0.00]} \\
& {[10.80,16.80,0.00] \quad[13.90,14.80,0.00]} \\
& {[15.30,13.60,0.00] \quad[15.40,13.60,0.00]} \\
& {[19.00,20.20,0.00] \quad[29.70,28.30,0.00]}
\end{aligned}
$$

$\begin{array}{ll}{[36.70,31.00,0.00]} & {[38.40,30.60,0.00]} \\ {[38.40,30.60,0.00]} & {[39.50,27.30,0.00]} \\ {[36.70,20.10,0.00]} & {[30.20,12.10,0.00]} \\ {[25.00,7.97,0.00]} & {[21.90,6.25,0.00]} \\ {[21.80,6.25,0.00]} & {[22.60,4.94,0.00]} \\ {[23.70,2.19,0.00]} & {[23.50,-2.46,0.00]} \\ {[23.70,-7.32,0.00]} & {[25.80,-14.00,0.00]} \\ {[25.40,-25.40,0.00]} & {[13.70,-34.30,0.00]} \\ {[4.27,-36.60,0.00]} & {[-0.12,-36.70,0.00]} \\ {[0.01,-36.70,0.00]} & \end{array}$

- The weight:

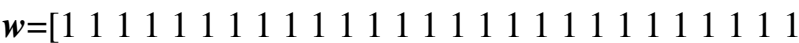

11111111111111111111111111 $11]$

- The knot vector:

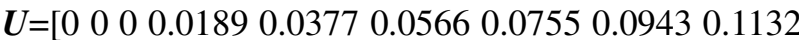

$\begin{array}{llllll}0.1321 & 0.1509 & 0.1698 & 0.1887 & 0.2075 & 0.2264\end{array}$

$\begin{array}{lllllll}0.2453 & 0.2642 & 0.2830 & 0.3019 & 0.3208 & 0.3396\end{array}$

$\begin{array}{lllllll}0.3585 & 0.3774 & 0.3962 & 0.4151 & 0.4340 & 0.4528\end{array}$

$\begin{array}{lllllll}0.4717 & 0.4906 & 0.5094 & 0.5283 & 0.5472 & 0.5660\end{array}$

$\begin{array}{lllllll}0.5849 & 0.6038 & 0.6226 & 0.6415 & 0.6604 & 0.6792\end{array}$

$\begin{array}{lllllll}0.6981 & 0.7170 & 0.7358 & 0.7547 & 0.7736 & 0.7925\end{array}$

$\begin{array}{lllllll}0.8113 & 0.8302 & 0.8491 & 0.8679 & 0.8868 & 0.9057\end{array}$

0.92450 .94340 .96230 .9811111 1]

- The order:

$p=2$

\section{Appendix B:}

The parameters of the NURBS surface in this work.

- The control points:

$\boldsymbol{P}=\{[-50,50,-4]$

$[-33.33333333333,50,-4][-16.66666666667,50,-6]$

$[0,50,-5][16.6666666666667,50,-6]$

$[33.3333333333333,50,-4][50,50,-4]$

$[-50,40,-6][-33.33333333333333,40,-6]$

$[-16.666666666667,40,0][0,40,-2]$

$[16.66666666667,40,0][33.333333333333,40,-6]$

$[50,40,-6][-50,20,-8]$

$[-33.33333333333,20,-8][-16.666666666667,20,-4]$

$[0,20,0][16.66666666667,20,-4]$

$[33.33333333333,20,-8][50,20,-8]$

$[-50,0,-10][-33.33333333333,0,-10]$

$[-16.66666666667,0,-6][0,0,0]$

$[16.66666666667,0,-6][33.33333333333,0,-10]$

$[50,0,-10][-50,-20,-6]$ 
$[-33.33333333333,-20,-6][-16.66666666667,-20,-2]$

$[0,-20,0][16.66666666667,-20,-2]$

$[33.33333333333,-20,-6][50,-20,-6]$

$[-50,-40,-4][-33.33333333333,-40,-8]$

$[-16.66666666667,-40,-8][0,-40,-2]$

$[16.666666666667,-40,-8][33.33333333333,-40,-8]$

$[50,-40,-4][-50,-50,-4]$

$[-33.33333333333,-50,-6][-16.66666666667,-50,-4]$

$[0,-50,-6][16.66666666667,-50,-4]$

$[33.33333333333,-50,-6][50,-50,-4]\}$

- The weight:

$\boldsymbol{W}=\{[1][1][2][2][2][1]$ [1];

\section{References}

1. Luu A-T, N-I Kim, and J Lee (2015) NURBS-based isogeometric vibration analysis of generally laminated deep curved beams with variable curvature. Compos Struct 119: 150-165.

2. Abbasnia A, and M Ghiasi (2014) Nonlinear wave transmission and pressure on the fixed truncated breakwater using NURBS numerical wave tank. Latin American Journal of Solids and Structures 11: 51-74.

3. Li X-W, Y-J Yang, W Zeng, Y-L Bi, J-L Xu, G Xu, and X-J Zhang (2022) Area-Preserving Hierarchical NURBS Surfaces Computed by the Optimal Freeform Transformation. Comput Aided Design 143: 103134.

4. Du X, and B Wang (2022) A C3-continuous NURBS transition scheme for the CNC machining of short linear segments. Precision Engineering 73: $1-10$.

5. Zhu L, B Yan, Y Wang, Y Dun, J Ma, and C Li (2021) Inspection of blade profile and machining deviation analysis based on sample points optimization and NURBS knot insertion. Thin-Walled Structures 162: 107540.

6. Zhang D, Z Wang, H Ling, and X Zhu (2021) Kriging-based shape optimization framework for blended-wing-body underwater glider with NURBS-based parametrization. Ocean Engineering 219: 108212 .

7. Yuan H, T Yu, and T Q Bui (2021) Multi-patch local mesh refinement XIGA based on LR NURBS and Nitsche's method for crack growth in complex cracked plates. Engineering Fracture Mechanics
[1] [1] [2] [2] [2] [1] [1];

[1] [1] [2] [2] [2] [1] [1];

[1] [1] [2] [2] [2] [1] [1];

[1] [1] [2] [2] [2] [1] [1];

[1] [1] [2] [2] [2] [1] [1];

[1] [1] [2] [2] [2] [1] [1]\}

- The knot vector:

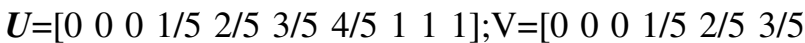

$4 / 5111]$;

- The order:

$p=2$;

$q=2$.

\section{0: 107780}

8. Zhou X, X Liu, M Li, Z Wang, and X Meng (2017) Post-processor development of a five-axis machine tool with optimization tool radius compensation. Int J Adv Manuf Tech 88: 1505-1522.

9. Coelho M, D Roehl, and K-U Bletzinger (2017) Material model based on NURBS response surfaces. Applied Mathematical Modelling 51: 574-586.

10. Liu Y, H Li, and Y Wang (2012) Realization of a 5-axis NURBS Interpolation with Controlled Angular Velocity. Chinese Journal of Aeronautics 25: 124-130.

11. Morlet L, C Gentil, S Lanquetin, M Neveu, and J-L Baril (2019) Representation of NURBS surfaces by Controlled Iterated Functions System automata. Computers \& Graphics: X 2: 100006.

12. Liang F, C Kang, and F Fang (2020) A smooth tool path planning method on NURBS surface based on the shortest boundary geodesic map. Journal of Manufacturing Processes 58: 646-658.

13. Neythalath N, A Søndergaard, and J A Bærentzen (2021) Adaptive robotic manufacturing using higher order knowledge systems. Automation in Construction 127: 103702.

14. Jiang J, Y Guo, Z Huang, Y Zhang, D Wu, and Y Liu (2021) Adjacent surface trajectory planning of robot-assisted tooth preparation based on augmented reality. Engineering Science and Technology, an International Journal. (in press)

15. Duong Q K, P Hubinsky, P Paszto, M Florek, J Sovcik, and M Kajan (2014) Effectiveness of Input Shaping and Real-Time Nurbs Interpolation for 
Reducing Feedrate Fluctuation. Procedia Engineering 96: 81-90.

16. Bingol O R, and A Krishnamurthy (2019) NURBS-Python: An open-source object-oriented NURBS modeling framework in Python. SoftwareX 9: 85-94.

17. Wang Y, X Xu, Z Zhao, W Deng, J Han, L Bai, X Liang, and J Yao (2021) Coordinated monitoring and control method of deposited layer width and reinforcement in WAAM process. Journal of Manufacturing Processes 71: 306-316.

18. Li J, Y Qiu, J Yang, Y Sheng, Y Yi, X Zeng, L Chen, F Yin, J Su, T Zhang, X Tong, and B Guo (2021) Effect of grain refinement induced by wire and arc additive manufacture (WAAM) on the corrosion behaviors of AZ31 magnesium alloy in $\mathrm{NaCl}$ solution. Journal of Magnesium and Alloys. (in press)

19. Duarte V R, T A Rodrigues, N Schell, R M Miranda, J P Oliveira, and T G Santos (2020) Hot forging wire and arc additive manufacturing (HF-WAAM). Additive Manufacturing 35: 101193.

20. Zeng Y, X Wang, X Qin, L Hua, and M Xu (2021) Laser Ultrasonic inspection of a Wire + Arc Additive Manufactured (WAAM) sample with artificial defects. Ultrasonics 110: 106273.

21. Al-Nabulsi Z, J T Mottram, M Gillie, N Kourra, and M A Williams (2021) Mechanical and X ray computed tomography characterisation of a WAAM 3D printed steel plate for structural engineering applications. Construction and Building Materials 274: 121700.

22. Ke W C, J P Oliveira, B Q Cong, S S Ao, Z W Qi, B Peng, and Z Zeng (2021) Multi-layer deposition mechanism in ultra high-frequency pulsed wire arc additive manufacturing (WAAM) of NiTi shape memory alloys. Additive Manufacturing102513.

23. Singh S, S k Sharma, and D W Rathod (2021) A review on process planning strategies and challenges of WAAM. Materials Today: Proceedings 47: 6564-6575.

24. Rodrigues $\mathrm{T}$ A, N Bairrão, F W C Farias, A Shamsolhodaei, J Shen, N Zhou, E Maawad, N Schell, T G Santos, and J P Oliveira (2022) Steel-copper functionally graded material produced by twin-wire and arc additive manufacturing
(T-WAAM). Materials \& Design 213: 110270.

25. Singh S R, and P Khanna (2021) Wire arc additive manufacturing (WAAM): A new process to shape engineering materials. Materials Today: Proceedings 44: 118-128.

26. Lefkopoulos V, and M Kamgarpour (2021) Trajectory planning under environmental uncertainty with finite-sample safety guarantees. Automatica 131: 109754.

27. Liu H, T B Gjersvik, and A Faanes (2022) Subsea field layout optimization (Part I) - directional well trajectory planning based on 3D Dubins Curve. Journal of Petroleum Science and Engineering 208: 109450.

28. Wang H, Q Zhao, H Li, and R Zhao (2021) Polynomial-based smooth trajectory planning for fruit-picking robot manipulator. Information Processing in Agriculture. (in press)

29. Piegl L, and W Tiller, 1997, The Nurbs Book, Springer-Verlag Berlin Heidelberg New York, New York.

30. RS H, and D J, 1965, Kinematic Synthesis of Linkages, McGraw-Hill, New York.

31. Xu C, Y. Li, X Zhou, E Jiao, and Y Liu (2013) Kinematics analysis and simulation of MOTOMAN-UP6 robot. Machine Tool \& Hydraulics 41: 144-149.

32. Wang Z Q, X Q Liu, X R Wang, C Y Li, N Yang, T S Lin, and P He (2021) Robotic milling of complex NURBS surface with fixed cutter axis control method. Industrial Robot 48: 413-422.

33. Wang Y S, X R Wang, Z Q Wang, T S Lin, and P He (2017) Preparation and microstructure of AlCoCrFeNi high-entropy alloy complex curve coatings. Mater Sci Tech 33: 559-566. 